\title{
EMPRENDIMIENTO, INNOVACIÓN, TECNOLOGÍAS DE LA INFORMACIÓN Y LA DE COMUNICACIÓN: CASO DE ESTUDIANTES DE ADMINISTRACIÓN DE EMPRESAS DE LA UNIVERSIDAD DE CUNDINAMARCA, COLOMBIA Y DE LA UNIVERSIDAD DE COLIMA, MÉXICO, EN LA CRISIS SANITARIA*
}

\begin{abstract}
Entrepreneurship, innovation, information and communication technologies: the case of Business Administration students from the University of Cundinamarca, Colombia and the University of Colima, Mexico, in the health crisis
\end{abstract}

\begin{tabular}{|c|c|}
\hline Fabio Orlando Cruz Páez iD & Cruz Osbaldo Sánchez Figueroa iD \\
\hline \multirow[t]{3}{*}{$\begin{array}{r}\text { Magíster en Administración de Organizaciones. Docente de } \\
\text { la Universidad de Cundinamarca. Colombia. } \\
\text { Correo electrónico: focruz@ucundinamarca.edu.co }\end{array}$} & $\begin{array}{r}\text { Doctor en Ciencias Administrativas y Negocios } \\
\text { Internacionales. Docente de la Universidad de Colima. } \\
\text { Correo electrónico: cruzfigueroa@ucol.mx }\end{array}$ \\
\hline & Camilo Andrés Jiménez Cuellar iD \\
\hline & $\begin{array}{r}\text { Administrador de Empresas, Universidad de } \\
\text { Cundinamarca, Colombia. } \\
\text { Correo electrónico: camiloajimenez@ucundinamarca.edu.co }\end{array}$ \\
\hline
\end{tabular}

Resumen

En el presente artículo de investigación se busca presentar un análisis de las variables del emprendimiento, de las Tecnologías de la información y la comunicación, de la innovación, de la financiación y de la inversión, respecto de la intención de emprender, teniendo como sujetos de análisis a los estudiantes del programa de Administración de Empresas de la Universidad de Cundinamarca en Colombia y a los estudiantes de ese mismo tipo de programa, en la Universidad de Colima en México. Para lo anterior, se tiene en cuenta la crisis económica actual, relacionada con la incidencia de la pandemia generada por el Covid-19. El tipo de investigación planteada es de carácter cuantitativo, con un alcance descriptivo; además, se sigue un enfoque no exploratorio, transeccional y de campo, para lo cual se lleva a cabo una recolección de datos por medio de encuestas, entendidas estas como un instrumento de investigación. Por su parte, los datos recolectados dejan ver un impacto relevante de las TIC en los procesos de emprendimiento innovador; además, se aprecia

\footnotetext{
*Proyecto de investigación del semillero de investigación GEICAEC, Universidad de Cundinamarca, en convenio con el programa de internacionalización de la Universidad de Colima - México, el cual lleva por título: "Análisis del emprendimiento de estudiantes de pregrado en el núcleo de conocimiento de Administración de Empresas en la Universidad De Cundinamarca, Colombia y la Universidad de Colima, México.
}

\footnotetext{
Cómo citar

Cruz-Páez, F, O.; Sánchez-Figueroa, C.O. y Jiménez-Cuellar, C.A. (2021). Emprendimiento, innovación, tecnologías de la información y la de comunicación: caso de estudiantes de administración de empresas de la Universidad de Cundinamarca, Colombia y de la Universidad de Colima, México, en la crisis sanitaria. Revista FACCEA, Universidad de la Amazonia, Vol. 11(1), 72-88.

DOI: https://doi.org/10.47847/faccea.v11n1a5
} 
el mínimo conocimiento por parte de los participantes, respecto de las oportunidades de financiación, y se observa una escasa intención hacia el emprendimiento de negocio, por parte, tanto de los estudiantes de la Universidad de Cundinamarca en Colombia, como de los estudiantes de la Universidad de Colima en México.

Palabras clave: emprendimiento, financiación, inversión, Tecnologías de la Información y la Comunicación.

\begin{abstract}
This research article aims to analyze the variables of entrepreneurship, information and communication technology, innovation, financing and investment regarding the intention to undertake by students of the business administration program of the Universidad de Cundinamarca in Colombia and the Universidad de Colima in Mexico, taking into account the current economic crisis in the incidence of Covid 19. The type of applied research is quantitative, with a descriptive scope, a non-exploratory, transectional and field approach, collecting data through the survey research instrument. The data collected show a relevant impact of ICT in innovative entrepreneurship processes, in addition, the minimum knowledge on the part of the participants in financing opportunities was appreciated, as well as the scarce intention to start a business on the part of students of the Universidad de Cundinamarca, Colombia and the Universidad de Colima, Mexico.
\end{abstract}

Key words: entrepreneurship, financing, investment, Information and Communication Technologies.

\title{
Introducción
}

Es notorio el debilitamiento que, desde la cultura organizacional, han tenido que asumir los empresarios en los procesos internos y su funcionamiento en el mercado, lo cual ha incidido en que los mismos debieran flexibilizarse; ello debido a la crisis sanitaria que ha afectado a toda la población mundial entre los años 2019 y 2020. En tal sentido, se puede entender que la actividad económica debe mejorar continuamente y que esta ha de permanecer en un proceso continuo de adaptación, como bien lo mencionaba Darwin en 1921, cuando publicó, el 24 de noviembre de 1859, su obra El origen de las especies, en la cual mencionaba que "las especies que sobreviven no son las más fuertes ni las más inteligentes, sino aquellas que se adaptan mejor al cambio". Teniendo en cuenta lo anterior, se entiende que la competitividad sugiere a las empresas un cambio que transforme el papel en el mercado nacional e internacional de los países (Llano, 2018).

Es así como se infiere en el planteamiento de la siguiente pregunta problematizadora: ¿Cómo influye entre los estudiantes del programa de Administración de Empresas de la Universidad de Cundinamarca Colombia y de la Universidad de Colima México, tanto las Tecnologías de la información y la comunicación, como la innovación social, la económica y la cultural, ¿en la intención de aquellos para emprender? Teniendo en cuenta lo anterior, el presente documento de 
investigación pretende conocer el nivel de importancia que se da al uso de las Tecnologías de la información y la comunicación, así como la que se da a la innovación social, económica y cultural, dentro del desarrollo y de la creación de emprendimientos, por parte de los estudiantes de administración de empresas en Colombia y en México en pro de la competitividad respecto a los cambios que se generan continuamente en el contexto empresarial y de los líderes empresarios; ello, específicamente durante una época que ha afectado en gran medida el proceder de las pequeñas, medianas y grandes empresas. Lo anterior, se analiza teniendo en cuenta que las variables señaladas, las cuales resultan vitales para el diario quehacer de los futuros profesionales, de acuerdo con las situaciones que se presentan en contextos globales, internacionales y locales, y que impactan las relaciones entre personas naturales y/o jurídicas-empresas.

Lo antes expuesto, teniendo en cuenta a su vez que la crisis sanitaria derivada de la enfermedad causada por el Covid-19 ha impulsado en el contexto social, empresarial y estatal, una invitación a la transformación y/o a la generación de cambios en las comunidades y en las empresas públicas y privadas, a lo largo de países como Colombia y México, cambios que deberían inferir en el desarrollo de la sociedad y del país. En tal sentido, desde Clacso (2020) se afirma por ejemplo que en general los países tienen condiciones desiguales en su estructura y que los sistemas de salud se encuentran de la misma forma bajo 30 años de neoliberalismo; ello especialmente en Estados débiles respecto a lo público, pero que están fortalecidos en pro de la privatización de productos y servicios en todos los sectores económicos, lo cual ha conducido a una mayor precarización laboral, además de oferta de los llamados catastróficos; todo lo anterior, financiado por el gasto de bolsillo que deben asumir las familias para solventar el gasto público.

Por su parte, el hecho de encontrarse en una época de crisis sanitaria, no se deja de lado las mínimas oportunidades de empleo a las que se enfrenta un estudiante en el transcurso y/o culminación de la carrera; por tanto, se pretende conocer la intención que los mismos tienen para emprender y para sacar provecho de las nuevas tendencias y oportunidades que el mercado ofrece.

\section{Emprendimiento}

El emprendimiento es una representación de pensar, razonar y actuar de manera racional tanto desde las oportunidades y las necesidades, a través de una visión global desarrollada a partir del liderazgo equilibrado y gestionada con un riesgo calculado, apreciado en la creación de valor que brinda beneficios a la empresa, la economía y la sociedad. (Fernández-Salinero y de la Riva, 2014, p.139). Es como de esta forma el emprendedor inicia acciones innovadoras, puesto que el empresario es quien se encuentra intrínsecamente unido a la entidad en cualquiera de los roles que este adopta ya sea propietario, socio o directivo. (Bucardo Castro, Saavedra García y Camarena Adame, 2015).

De acuerdo con la acción de emprender de las personas, la contribución que pretende generarse en las nuevas y pequeñas empresas frente a la empleabilidad intraemprendedora y al emprendimiento se brinda desde la implementación de innovaciones, ya que según Cruz, Mera, \& Lechuga (2019) "la 
estrategia empresarial que muestra a los mejores emprendedores son las generadas a partir de propuestas que nacen de ideas innovadoras que se gestionan y ejecutan permitiendo fortalecer el desarrollo empresarial y de la sociedad" (p. 188). Esto quiere decir que en definitiva se impacta al desarrollo económico, cultural y social, siendo algunos de los motivos que justifican el progresivo interés de instituciones académicas, gubernamentales, empresarios, y población en general, hacia las iniciativas emprendedoras. También se ha relacionado el emprendimiento con el descubrimiento de oportunidades rentables. Shane \& Venkataraman (2000) afirman que las oportunidades de emprendimiento son escenarios en los que los bienes, los servicios, las materias primas, los métodos y las metodologías de organización se pueden vender e introducir a un precio mayor que el del costo de producción (p. 220).

El emprender es un tema del que se habla hoy en día de manera recurrente, dada la necesidad de superación de muchas personas, quienes buscan lograr una oportunidad y tener un desarrollo personal, generando resultados importantes de acuerdo con el esfuerzo realizado; aunque también se pueden generar resultados decepcionantes, motivados por la poca información y motivación. Al centrase específicamente en la innovación y en el Emprendimiento, también se hace referencia al liderazgo, a la inversión, a la constancia, a los proyectos, a los desafíos y a las oportunidades; entre otras palabras relacionadas con la idea de emprender e innovar. Corredor (2007) citado en Vélez \& Ortiz (2016) afirma que en los últimos años, las teorías asociadas al emprendimiento y a la innovación determinaron que la función del emprendedor es determinante en los procesos de innovación (p. 349).

Ahora bien, teniendo en cuenta los emprendimientos y los emprendedores que logran consolidar empresas de acuerdo con elconocimiento adquirido desde la universidad y que logran fortalecer la experiencia empírica, se muestra que las empresas juegan un papel importante en la innovación de procesos, de procedimientos y a nivel organizacional, desde la innovación aplicada por los líderes y por las empresas. Así, de acuerdo con lo que afirman Cruz, Ibarra, Rueda y Olivares (2020), en los países de América Latina se evidencia una gran necesidad de incursionar de manera contundente en la innovación; ello, desde la innovación social, tanto por parte del gobierno como por parte de las empresas, de la universidad y de la sociedad, lo anterior, a partir del aporte que brinda la responsabilidad social empresarial en los actuales contextos globalizados.

\section{Tecnologías de la información y la comunicación}

Las Tecnologías de la información y la comunicación (TIC), comprenden como concepto los servicios usados para intercambiar información como el correo electrónico, foros, redes sociales, productos audiovisuales y, en general buscadores de información; igualmente comprende las redes de telecomunicaciones que los soportan, entre ellas el internet, la intranet corporativa, la telefonía fija y móvil y los equipos que facilitan el acceso como lo son los ordenadores personales, los celulares, las tabletas, los reproductores de audio y de video (Altés. 2013) A su vez, dichas herramientas son consideradas de gran importancia para los emprendedores, los empresarios y las empresas, en el uso de las TIC. 
El uso de internet determina el nivel de importancia que existe en cuanto al uso de tecnologías de la información y la comunicación, representado en las nuevas tecnologías, respecto de la innovación social, económica y cultural que se da en contextos empresariales y personales. Como lo indican diferentes autores, internet supone un salto cualitativo de gran magnitud, que además cambia y redefine los modos de conocer y de relacionarse de los seres humanos (Ayala \& Gonzales, 2015, p. 27). Lo anterior puede determinar su importancia en el impacto para el fortalecimiento de las empresas; para el nacimiento de otras y a su vez, para la innovación. Lo anterior también hace referencia a que el término innovación va más allá de la tecnología y abarca, a grandes rasgos, aspectos como la creatividad, así como la manera y la forma en que se pretende hacer llegar un producto o servicio al cliente. López Pérez (2012) sugiere algunas formas de conseguir este resultado generando satisfacción a nuevas necesidades de los clientes, consiguiendo nuevas formas de obtener beneficios, o generando valor de una forma poco convencional (p. 93), lo cual debe estar en función de una adaptación a las nuevas formas de comercializar los productos y/o servicios.

Lo anterior se relaciona también con el sistema de las tecnologías de la información y la comunicación, las cuales evidentemente están provocando un cambio económico y social; por ende, su adecuado uso puede generar ventajas respecto de los objetivos que se tracen. No cabe duda de que, en ámbito educativo, las TIC han permitido la eliminación de barreras espaciotemporales, facilitando la generación colectiva de conocimiento (Gairín, citado en Hernández, 2017, p. 129). Si se analiza bien, las TIC están influyendo en las nuevas formas de emprender y de satisfacer las necesidades de los clientes, logrando romper paradigmas, pues permiten configurar nuevas formas de interacción entre los diferentes agentes dentro del ámbito empresarial y comercial.

Con lo anterior ha quedado demostrado que, al analizar las variables de la tecnología de la información y la comunicación, la innovación social, económica y cultural y su influencia en la intención de emprender de los estudiantes del programa de Administración de Empresas de la Universidad de Cundinamarca en Colombia y de la Universidad de Colima en México, teniendo en cuenta la crisis económica actual es parte esencial del uso y potencialización de cada una de ellas respecto a la competencias de los futuros profesionales.

Así, se puede inferir que la variable de las tecnologías muestra que ellas pueden ser especializadas para el sector económico real ya que ofrecen aplicaciones específicas para procesar la información y la toma de decisiones con gestión avanzada, puesto que se da a través de procesos empresariales, en el contexto de negociaciones internacionales (Cruz Páez y Vanegas Flórez, 2020) como se da en la relación empresarial de profesionales de las dos Universidades.

\section{Financiación}

La financiación es un factor clave para el emprendimiento, especialmente en periodos de crisis, en 
tanto la restricción financiera dificulta más el acceso al crédito dirigido hacia los nuevos proyectos empresariales, relacionados con las pequeñas y las medianas empresas (Santero Sánchez, de la Fuente-Cabrero y Laguna Sánchez, 2016)

Entre las oportunidades que se presentan se encuentra la financiación empresarial o también aquello que se conoce como capital de riesgo, los cuales son aportes de dinero y de valor agregado realizados por inversionistas individuales o por instituciones, con el fin de obtener un rápido crecimiento para las pequeñas empresas y con la intención de buscar significativamente el incremento de su valor. En el estudio de Fracica Naranjo (2009) se especifica que los inversionistas realizan importantes aportes de valor agregado, tales como asesoría estratégica, operativa, financiera, de mercadeo, marco legal y contactos comerciales y financieros; a esto también se le conoce como "dinero inteligente" (p. 37). Esta inyección de capital termina siendo considerada como una clave fundamental, sobre todo para emprendedores e innovadores, la cual, al mismo tiempo, genera un dinamismo en el ámbito empresarial de la economía, convirtiéndola en más productiva.

\section{Inversión}

Las inversiones facilitan el emprendimiento de las diferentes comunidades, como los son emprendedores empíricos y los emprendedores en formación profesional o profesionales graduados. Básicamente, para encontrar la manera de emprender es necesario obtener inversiones de impacto en las futuras empresas y en las ya consolidadas. Contreras, Pedraza y Martínez (2017) definen estas inversiones como la colocación rentable de recursos financieros, en iniciativas empresariales en pro del desarrollo sostenible de la sociedad.

En la actualidad, a las personas que tienen intenciones de emprender, pero que no cuentan con el apoyo de alguien que tenga experiencia en el ámbito empresarial y en el desarrollo de $\mathrm{s}$ emprendimientos, que a su vez no cuentan con recursos ni con conocimientos, les es difícil comenzar esta actividad; así lo evidencia Valencia Herrera (2015) en tanto considera que financiar la inversión, respecto de los costos de las transacciones y de la información que incrementan el costo de la fuente de financiación, es proveniente de fuentes internas, de las utilidades que se retuvieron y posteriormente de fuentes externas, como de deudas y emisiones de capital.

En general, para este tipo de población sin formación empresarial es difícil alcanzar el objetivo del emprendimiento; por ello, para los futuros profesionales, en especial para los que cuentan con núcleos básicos de conocimiento en administración, resulta vital estructurar competencias a partir de las cuales aquellos fortalezcan sus conocimientos, habilidades y destrezas empresariales y además se apoyen con las organizaciones que promueven la incubación y la aceleración empresarial, organizaciones que a su vez tienen un papel fundamental y un relevante significado para los emprendedores.

Johnson y Lee (2013) citado en Contreras, Pedraza y Martínez (2017), afirman que una inversión puede ser gestionada en negocios, bien sea por parte de corporaciones, de proyectos o por parte del 
gobierno u otro ente que ofrezca una solución con potencial rentable y orientación dirigida hacia mercados específicos, que tengan un mayor impacto y que cuenten con retos sociales y/o ambientales, que el inversor desee ayudar a gestionar.

Es en el mencionado ámbito en el cual las empresas, las organizaciones, las universidades o cualquier otro tipo de entidad, deben brindar su apoyo a partir de acciones como talleres, cursos, capital, relaciones con empresas, clientes, asesoramiento, etc. Cada organización aporta de distintas maneras evidenciando interés en el proyecto, en tanto se entiende que todo tipo de apoyo puede resultar de gran ayuda para los emprendedores.

Por otra parte, se infiere que pueden gestionarse diversas figuras financieras para los emprendimientos, entre ellas las que permiten adquirir créditos, bonos de deuda, deudas convertibles como mezzanine y/o inversiones directas para ejecutar operaciones, con la finalidad de invertir en las empresas (Johnson y Lee, 2013 citado en Contreras Pacheco, Pedraza Avella, Martínez Pérez, 2017).

\section{Metodología}

La investigación que se presenta en este artículo se realizó siguiendo algunos parámetros de Gómez, Sánchez y Tovar (2018), a partir de un enfoque cuantitativo, con una metodología cuantitativa de tipo descriptivo; así mismo, se adelantó siguiendo un diseño no experimental, transeccional y de campo. Las variables que fueron tratadas y aplicadas por medio de una encuesta son las siguientes: el emprendimiento, las tecnologías de la información y la comunicación, la financiación, la inversión y la innovación. Lo anterior, con el objetivo de analizar la intención de emprender por parte de los estudiantes de Administración de Empresas de la Universidad de Cundinamarca en Colombia, y de la Universidad de Colima en México.

Se establece que el enfoque de la investigación es cuantitativo, por lo cual se entiende que la investigación debe ser objetiva y que el fenómeno observado o medido no deberá ser afectado por el investigador, evitando influencias de aquél en los resultados del estudio o interferencias en los procesos, y que los resultados no se alteren por las tendencias de otros investigadores (Unrau, Grinnell y Williams, 2005 citado en Hernández Sampieri, Fernández Collado, \& Baptista Lucio, 2014). La investigación también es cuantitativa en el modelo de investigación frente a la recolección de datos, lo cual se considera de vital importancia para los estudios que se realizan, pues a través de este tipo de investigación los datos se validan tanto interna como externamente (Hernández Sampieri, Fernández Collado, \& Baptista Lucio, 2014).

Po otra parte, el alcance establecido es descriptivo, puesto que se indagan los acontecimientos en las categorías de las variables en la población estudiada (Hernández Sampieri, Fernández Collado, \& Baptista Lucio, 2014). 
A su vez, este análisis investigativo que tiene como sujetos de estudio a los estudiantes de Administración de Empresas de las universidades de Cundinamarca en Colombia y de Colima en México tiene un diseño no experimental, ya que Hernández, Fernández, \& Baptista (2014) afirman que las investigaciones adelantadas de este modo, se realizan sin manipular las variables y únicamente observando los fenómenos en el ambiente natural, con el fin de analizar posteriormente cada hecho o fenómeno indagado. Así, en el presente estudio se analizaron las variables del instrumento de manera natural sin afectarlas.

Por su parte el diseño de investigación elegido es el transversal, ya que en el estudio que se gestionó no se le realizará un seguimiento, debido a que además se efectuó en un periodo explícito (Hernández Sampieri, Fernández Collado, \& Baptista Lucio, 2014).

Finalmente, las variables que se permite describir en la investigación con base en su resultado son las siguientes: el emprendimiento, la tecnología de la información y la comunicación, la innovación, la financiación y la inversión. Estas representan los 5 pilares que permiten dar respuesta al objetivo, a partir de conocer los resultados del análisis del instrumento aplicado, el cual tiene un total de 21 preguntas.

\section{Participantes}

La muestra se determinó a partir del universo de estudiantes de Administración de Empresas de la Universidad de Cundinamarca en Colombia y de la Universidad de Colima en México; para la misma se consideraron inicialmente estudiantes de todos los semestres de las dos Universidades, los cuales sumaron en total 1223 estudiantes como criterio de inclusión. A partir de una muestra no probabilística se determinaron 166 estudiantes seleccionados, de los cuales, finalmente se eligió un total de 158 estudiantes de las dos universidades; esto quiere decir que del 95\% del universo escogido, se da un error de $5 \%$, considerándose por tanto una muestra confiable para la recolección de los datos. Dicha recolección se realizó de manera electrónica, a partir de un formulario de Google docs; la encuesta estuvo abierta durante los meses de mayo a junio de 2020. De los 158 participantes, el 61\% ( $n=97)$ pertenecían a la Universidad de Cundinamarca (Colombia), y el 39\% $(n=61)$ a la Universidad de Colima (México). La edad promedio de quienes más contestaron la encuentra oscila entre los 21 a los 29 años; lo anterior con porcentajes del $65 \%(n=103)$ y de 200 menos años 35\% ( $n=55)$. La selección de los estudiantes encuestados se llevó a cabo con base en las características de perfil del programa académico del estudiante y de la ubicación geográfica.

\section{Discusión y resultados}

Con base en los resultados de la encuesta, la cual fue aplicada a 158 estudiantes, se tienen los siguientes hallazgos, a partir de los cuales se realizó un análisis por medio del programa Excel a través de tablas dinámicas, las cuales fueron usadas para obtener datos estadísticos, con el fin de analizarlos de manera pormenorizada. 
La encuesta se dividió en los cinco bloques siguientes: preguntas filtro; medición del emprendimiento; opinión respecto a las TIC; innovación; financiación e inversión. Con relación a las preguntas filtro establecidas, de los encuestados, el 100\% cumple la característica establecida, es decir, son estudiantes de administración de empresas. Entre los participantes se encuentran $36 \%$ $(n=95)$ hombres y 64\% $(n=166)$ mujeres (Tabla 1).

Tabla 1.

Género y edad de los encuestados.

\begin{tabular}{lccc}
\hline Género y edad & H & M & Total general \\
\hline Universidad de Cundinamarca (Colombia) & 61 & 111 & 172 \\
20 años o menos & 7 & 15 & 22 \\
$21-29$ años & 54 & 96 & 150 \\
Universidad de Colima (México) & 34 & 55 & 89 \\
20 años o menos & 14 & 19 & 33 \\
$21-29$ años & 20 & 36 & 56 \\
Total general & 95 & 166 & 261 \\
\hline
\end{tabular}

Fuente: elaboración propia. (2020).

\section{Emprendimiento}

En la tabla en la cual se consignan los resultados de la medición del nivel de emprendimientos generados por los estudiantes se observa que, a partir de octavo semestre, hay un $11 \%(n=17)$ de estudiantes que tienen emprendimientos propios o familiares; esta es la cifra más alta en comparación con las de otros semestres; por su parte, se encuentra que el $49 \%$ ( $n=77)$ del total de los encuestados no tiene emprendimientos o no son emprendedores.

Tabla 2.

Nivel de emprendimiento.

\begin{tabular}{ccccc}
\hline Semestre al que pertenece & No tienen & Propia & Familiar & Total general \\
\hline 1 & $1 \%$ & $3 \%$ & $1 \%$ & $4 \%$ \\
2 & $9 \%$ & $8 \%$ & $3 \%$ & $19 \%$ \\
3 & $0 \%$ & $1 \%$ & $0 \%$ & $1 \%$ \\
4 & $7 \%$ & $1 \%$ & $0 \%$ & $8 \%$ \\
5 & $4 \%$ & $8 \%$ & $2 \%$ & $13 \%$ \\
6 & $5 \%$ & $4 \%$ & $2 \%$ & $11 \%$ \\
7 & $4 \%$ & $3 \%$ & $1 \%$ & $8 \%$ \\
8 & $13 \%$ & $7 \%$ & $4 \%$ & $23 \%$ \\
9 & $6 \%$ & $2 \%$ & $1 \%$ & $9 \%$ \\
10 & $1 \%$ & $1 \%$ & $2 \%$ & $4 \%$ \\
\hline Total general & $49 \%$ & $37 \%$ & $15 \%$ & $100 \%$ \\
\hline
\end{tabular}

Fuente: elaboración propia. (2020). 


\section{Emprendimiento}

De acuerdo con los resultados obtenidos que se observan en la Tabla 3, el 100\% ( $n=158)$ de los estudiantes consideran positivo el desarrollo de capacidades emprendedoras, precisamente en la época de cambio analizada. Además, gracias al estudio se encuentra que las razones más importantes por las cuales se considera relevante adquirir estas competencias, están relacionadas con desarrollo de nuevas ideas innovadoras, que permitan aportar a la resolución de los problemas propios de la población, ello con un resultado de 30\% ( $n=47)$; a su vez, el $27 \% \quad(n=42)$ de los estudiantes consideran que es necesario evolucionar ante los cambios externos y de oportunidad; por otra parte, el $20 \%$ ( $n=31$ ) considera que es necesario obtener habilidades para el desarrollo de un negocio, con el objetivo de ser competitivo.

Tabla 3.

Aspectos positivos en el desarrollo del emprendedor.

\begin{tabular}{lr}
\hline \multicolumn{1}{c}{ Detalle } & $\%$ \\
\hline 1= Desarrollo de nuevas ideas innovadoras que permitan aportar a la resolución de & $30 \%$ \\
problemas de la población. & \\
$2=$ Es mejor depender de tu propio ingreso & $6 \%$ \\
$3=$ Genera oportunidades de empleo & $6 \%$ \\
4= Contribuye al desarrollo de la economía nacional & $9 \%$ \\
5= Es necesario obtener habilidades para el desarrollo de un negocio y ser competitivo & $20 \%$ \\
6= Es necesario evolucionar ante los cambios externos y de oportunidad & $27 \%$ \\
$7=$ No es conveniente debido a la situación presentada por el COVID-19 & $1 \%$ \\
\hline Total & $100 \%$ \\
\hline Fuente: elaboración propia. (2020).
\end{tabular}

Fuente: elaboración propia. (2020).

\section{Tecnologías de la información y la comunicación}

La Tabla 4 permiten evidenciar el conocimiento de casos de éxito de emprendimientos cercanos por parte de los encuestados. Se establece que el resultado fue de un $65 \%(n=103)$ de estudiantes que no conocen el inicio de emprendimientos o el crecimiento de proyectos de emprendimiento; solo un $35 \%$ ( $n=55)$ de los encuestados responde que sí ha sido testigo de casos de éxito en materia de emprendimientos.

Tabla 4.

Conocimiento de casos de éxito.

\begin{tabular}{lccc}
\hline Universidad a la que pertenece & Si & No & Total general \\
\hline Universidad de Cundinamarca & $16 \%$ & $46 \%$ & $61 \%$ \\
Universidad de Colima & $19 \%$ & $20 \%$ & $39 \%$ \\
\hline Total general & $35 \%$ & $65 \%$ & $100 \%$ \\
\hline Fuente: elaboración propia. (2020). & & &
\end{tabular}




\section{Tecnologías de la información y la comunicación}

El 94\% ( $n=149$ ) de los estudiantes que comprenden el rango de edad de entre los 21 a los 29 años, los cuales a su vez corresponden al $65 \%(n=103)$ de la totalidad de muestra, consideran necesaria la utilización de recursos tecnológicos. A su vez, el $42 \%(n=66)$ de los estudiantes tiene un buen dominio de las habilidades en el manejo de las TIC, y un 12\% ( $n=19)$ tiene un nivel excelente en esa misma competencia. Lo anterior permite identificar una buena adaptabilidad en lo que se refiere a la implementación de las tecnologías de la información y la comunicación, en procesos de emprendimiento.

\section{Tabla 5.}

El dominio de habilidades. Manejo de las TIC.

\begin{tabular}{lccccc}
\hline Edad & Nulo & Suficiente & Bueno & Excelente & Total general \\
\hline 20 o menos & $0 \%$ & $9 \%$ & $21 \%$ & $4 \%$ & $35 \%$ \\
$21-29$ & $1 \%$ & $11 \%$ & $42 \%$ & $12 \%$ & $65 \%$ \\
\hline Total general & $1 \%$ & $20 \%$ & $63 \%$ & $16 \%$ & $100 \%$ \\
\hline Fuente: elaboración propia. (2020). & & & &
\end{tabular}

Tabla 6.

Importancia merece la utilización de recursos tecnológicos, como apoyo a los procesos de emprendimiento.

\begin{tabular}{lc}
\hline Detalle & $\%$ \\
\hline $1=$ Necesario & $94 \%$ \\
$2=$ Opcional & $5 \%$ \\
$3=$ No aplica a los procesos de emprendimiento. & $1 \%$ \\
\hline Total & $100 \%$ \\
\hline Fuente: elaboración propia. (2020).
\end{tabular}

\section{Inversión}

Por otra parte, el $94 \% \quad(n=149)$ de los estudiantes considera beneficioso asociarse con inversionistas, con el fin de tener crecimiento en los proyectos de emprendimiento; el $55 \%$ ( $n=87$ ) piensa que existe una colaboración, asesoramiento y beneficios mutuos; el $27 \%(n=43)$ de los estudiantes opina que es necesario contar con fuentes de financiamiento y el $10 \%(n=16)$ considera que resulta beneficioso ya que es más corto el periodo de tiempo en el que se pueden lograr los objetivos (Tabla 7).

\section{Financiación}

Los estudiantes evidencian un conocimiento leve acerca de las fuentes de financiación (Tabla 8) que ofrece su entorno; en lo referente al conocimiento sobre las ayudas del gobierno se tiene una votación del $20 \%$ ( $n=91$ ); con respecto al financiamiento gubernamental se tiene un porcentaje de un $18 \%(n=79)$; sobre las fuentes útiles como lo son el capital semilla se tiene un $11 \%(n=48)$ del porcentaje de conocimiento y sobre el crowdfunding se tiene un $6 \%(n=27)$ del porcentaje. 
Tabla 7.

Justificaciones para asociarse.

\begin{tabular}{lc}
\hline Detalle & $\%$ \\
\hline 1= Es más corto el periodo de tiempo en el que se pude lograr estos objetivos. & $10 \%$ \\
2= Existe una colaboración, asesoramiento y beneficios mutuos & $55 \%$ \\
$3=$ Es necesario contar con fuentes de financiamiento. & $27 \%$ \\
$4=$ Analizar primero su influencia para luego decidir una posible sociedad & $1 \%$ \\
$5=$ No tiene desarrollada la idea & $2 \%$ \\
6=No le interesa o no se siente preparado & $4 \%$ \\
\hline Total & $100 \%$ \\
\hline
\end{tabular}

Fuente: elaboración propia. (2020).

Tabla 8.

Fuentes de financiación que conocen.

\begin{tabular}{lc}
\hline Detalle & $\%$ \\
\hline $1=$ Triple F (Family, Friends and Fools). & $4 \%$ \\
$2=$ Crowdfunding. & $6 \%$ \\
$3=$ Capital semilla o ángeles inversionistas. & $11 \%$ \\
$4=$ Venture capital (Capital de riesgo). & $2 \%$ \\
$5=$ Financiamiento Gubernamental. & $18 \%$ \\
$6=$ Concursos. & $11 \%$ \\
$7=$ Incubadoras y aceleradoras. & $9 \%$ \\
$8=$ Banca. & $16 \%$ \\
$9=$ Fondos de la Alianza del Pacífico. & $3 \%$ \\
$10=$ Gobierno. & $20 \%$ \\
\hline Total & $100 \%$ \\
\hline
\end{tabular}

Fuente: elaboración propia. (2020)

\section{Innovación}

La innovación también se ve reflejada en el nivel de participación de las universidades en el ámbito del emprendimiento. En la Tabla 9 se observa que el 33\% ( $n=52)$ de los estudiantes de la Universidad de Cundinamarca sienten que dicha institución, debería implementar más apoyo a la actividad emprendedora; a su vez, el 20\% (n=32) de los estudiantes de la Universidad de Colima creen que el apoyo de su institución es importante para la ejecución de ideas y para creación de empresa.

Debido a la contingencia experimentada en el momento en el que se escribe el presente trabajo, la cual es ocasionada por la pandemia derivada del Covid-19, se considera un espacio para evaluar la importancia que le atribuyen los estudiantes a la implementación y al uso de las TIC, en proyectos de emprendimiento en pequeñas empresas; lo anterior, debido a las restricciones y a los inconvenientes que surgieron para poder abrirlos negocios; en tal sentido, se observa que el $94 \%$ $(n=149)$ de la muestra considera que la coyuntura es un factor de oportunidad para adaptarse al 
Tabla 9.

Participación de su institución Universitaria en la actividad emprendedora.

\begin{tabular}{lcccc}
\hline $\begin{array}{l}\text { Universidad a la que } \\
\text { pertenece }\end{array}$ & $\begin{array}{c}\text { El apoyo es visible } \\
\text { y fácilmente } \\
\text { identificable. }\end{array}$ & $\begin{array}{c}\text { El apoyo es importante } \\
\text { para la ejecución de ideas más apoyo a la actividad } \\
\text { y creación de empresa. }\end{array}$ & $\begin{array}{c}\text { Debería implementar } \\
\text { emprendedora. }\end{array}$ & $\begin{array}{c}\text { Total } \\
\text { general }\end{array}$ \\
\hline Universidad de Cundinamarca & $8 \%$ & $20 \%$ & $33 \%$ & $61 \%$ \\
Universidad de Colima & $9 \%$ & $20 \%$ & $9 \%$ & $39 \%$ \\
\hline Total general & $18 \%$ & $41 \%$ & $42 \%$ & $100 \%$ \\
\hline
\end{tabular}

Fuente: elaboración propia. (2020).

Tabla 10.

Implementación de las TIC en la época del Covid-19. (Considera que la implementación de las TIC en emprendimientos con la contingencia vivida actualmente por el COVID-19).

\begin{tabular}{lcccc}
\hline $\begin{array}{l}\text { Universidad a la que } \\
\text { pertenece }\end{array}$ & $\begin{array}{c}\text { Una desventaja } \\
\text { debido a que no } \\
\text { pueden llegar a su } \\
\text { público objetivo. }\end{array}$ & $\begin{array}{c}\text { Un factor de oportunidad } \\
\text { para adaptarse al mundo } \\
\text { moderno con nuevos } \\
\text { productos o servicios. }\end{array}$ & $\begin{array}{c}\text { Una desventaja } \\
\text { por el mínimo } \\
\text { conocimiento de } \\
\text { empleo de las TIC. }\end{array}$ & Total general \\
\hline Universidad de Cundinamarca & $2 \%$ & $58 \%$ & $1 \%$ & $61 \%$ \\
Universidad de Colima & $1 \%$ & $36 \%$ & $1 \%$ & $39 \%$ \\
\hline Total general & $3 \%$ & $94 \%$ & $3 \%$ & $100 \%$ \\
\hline
\end{tabular}

Fuente: elaboración propia. (2020).

mundo moderno, con nuevos productos o servicios; solo el $3 \%(n=4)$ siente que esta es una desventaja, dado el escaso conocimiento en el uso de las TIC.

Por su parte, dentro el grupo de estudiantes que fueron encuestados, el $58 \%$ de la Universidad de Cundinamarca y el $36 \%$ de la Universidad de Colima considera que juega como factor de mayor relevancia, la oportunidad de adaptarse al mundo moderno, con productos y/o servicios, a partir de la gestión e implementación de las TIC; también se encuentra un resultado del 3\% en las dos Universidades, tanto para el factor de desventaja por no facilitarse llegar al público objetivo, como para el factor de desventaja por el mínimo conocimiento de las TIC.

Así bien, como aporte de la investigación se puede mencionar que los resultados reveladores, con base en los dos grupos que fueron analizados, esto es, los estudiantes de la Universidad de Colima y los estudiantes de la Universidad de Cundinamarca, son los siguientes:

En primera instancia, se encuentra que desde el ámbito emprendedor generado por parte de los estudiantes de los programas de Administración de Empresas, los resultados no fueron los más favorables, ya que existe un porcentaje negativo, con el $49 \%(n=77)$, de entre quienes tienen poca 
intención por emprender; esta intención se debería transformar a partir de la ruptura de paradigmas y considerando aportar más a la economía nacional y a la sociedad, por medio de la innovación y de la implementación de las TIC. Estas a su vez evidencian un papel importante para el crecimiento y para el fortalecimiento de las empresas. Como lo indica Cruz, Mera, \& Lechuga (2019), la innovación, como estrategia empresarial, determina que los mejores emprendedores son aquellos que generan propuestas que nacen de ideas que se hacen realidad, permitiendo fortalecer el desarrollo empresarial y el desarrollo de la sociedad (p. 188); de esa manera es posible a su vez mantener un nivel de competitividad alto, adaptándose a las nuevas necesidades de los clientes.

En tal sentido, lo anterior se puede considerar como un área de oportunidad para las empresas y para el gobierno, por lo cual, a partir de dichos estamentos se debería impeler a la implementación de estrategias que aporten al desarrollo de programas de emprendimiento, además de impulsar mecanismos que ayuden a eliminar los paradigmas mentales de los jóvenes que no se atreven a intentar emprender.

Por otra parte, a pesar de tener varias posibilidades de fuentes de financiación (economía y cultura) como las siguientes: el Centro de Innovación Tecnología y Gestión Organizacional (CITGO), entidad que ofrece atención, asesoría y consultoría en la Universidad de Cundinamarca en Colombia (Murcia, 2018, p. 15); el Fondo Emprender, el cual está disponible con fondos de capital semilla (Fondo Emprender, 2020); la Secretaría de Economía; el Programa Nacional de Emprendedores en México, que tiene como objetivo promover e impulsar en los mexicanos la cultura y el desarrollo empresarial para la creación de más y mejores emprendedores, empresas y empleos (Secretaria de Economía, 2010); el Nafin del programa de apoyo a emprendedores mexicanos, el cual busca apoyar tanto a empresas nuevas como empresas ya establecidas, que cuenten con proyectos productivos innovadores (Nafin, 2008); entre otras desde las cuales se pretende apoyar la actividad de emprender de manera eficaz, los estudiantes no cuentan con la información adecuada sobre los fomentos al emprendimiento. Esto demuestra que existe un problema de iniciativa que estanca a los emprendedores, asunto que se ve reflejado en la muestra; ello, en tanto un máximo del $20 \%$ ( $n=91$ ) de la muestra conoce acerca de las ayudas del gobierno. Así las cosas, esto último se ha de considerar como un aspecto bastante importante, ya que las oportunidades de crecer se encuentran disponibles para cualquier idea innovadora que aporte a los demás.

\section{Conclusiones}

El estudio aquí presentado a partir de las encuestas realizadas a los estudiantes de Administración de Empresas de la Universidad de Cundinamarca en Colombia y de la Universidad de Colima en México, en el cual se analizaron los factores de emprendimiento, tecnologías de la información y la comunicación, innovación, financiación e inversión, son aquí considerados como asuntos que deben ser fortalecidos por parte de los emprendedores; ello, a partir de la implementación de una educación continua o de extensión, que se fomente a través de los programas de emprendimiento y 
de empresarismo de las dos universidades señaladas, y desde actividades que generen un mayor impacto para la creación y/o aceleración de los emprendimientos, en los cinco pilares arriba expuestos. Lo anterior, en tanto se considera que los mismos tienen un aspecto significativo respecto de los cambios que trae consigo la época actual; misma que está incentivando el emprendimiento, en la medida en la que la crisis sanitaria ha afectado el desarrollo económico a nivel general dentro de los Estados y de los países, y en específico, respecto del gasto familiar y del gasto público.

Así las cosas, las universidades de Cundinamarca en Colombia y de Colima en México deberán realizar estudios empíricos, respecto de las competencias financieras y de innovación de los futuros profesionales; ello, en función de implementar un asertivo manejo de las TIC, y en la búsqueda, tanto de fomentar la creación de empresa, como de generar un fortalecimiento empresarial; ello, en pro del desarrollo organizacional y social.

Finalmente, se recomienda que en los diferentes programas de las dos universidades antes mencionadas se realice una investigación que permita analizar la capacidad de emprender de los estudiantes de las diferentes disciplinas o carreras universitarias, con el fin de comprender las competencias de gestión para el emprendimiento de futuros profesionales.

\section{Referencias bibliográficas}

Altés, J. (2013). Papel de las tecnologías de la información y la comunicación en la medicina actual. Seminarios de la Fundación Española de Reumatología, 14(2), 31-35. DOl: 10.1016/j.semreu.2013.01.005

Ayala, E. E., \& Gonzales, S. R. (2015). Tecnologías de la información y la Comunicación. Fondo Editorial de la UIGV. http://repositorio.uigv.edu.pe/bitstream/handle/20.500.11818/1189/Libro\%20TIC\%20\%282\%29-176\%20\%281\%29.pdf?sequence=1\&amp\%3Bamp\%3Bamp \%3BisAllowed=y

Bucardo Castro, A., Saavedra García, M. L. y Camarena Adame, M. E. (2015). Hacia una comprensión de los conceptos de emprendedores y empresarios. Suma de negocios, 6(13), 98-107. https://www.elsevier.es/es-revista-suma-negocios-208-articulo-hacia-una-comprension-conceptosemprendedores-S2215910X15000154

Contreras-Pacheco, O. E., PedrazaAvella, A.C. y Martínez Pérez, M. J. (2017). La inversión de impacto como medio de impulso al desarrollo sostenible: una aproximación multicaso a nivel de empresa en Colombia. Estudios Gerenciales, 33(142). DOI: 10.1016/j.estger.2017.02.002

Clacso. (2020). La crisis sanitaria del Covid-19. Grupo de Trabajo Estudios sociales para la salud del Consejo Latinoamericano de Ciencias Sociales. CLACSO. https://www.clacso.org/pronunciamiento-del-grupo-detrabajo-estudios-sociales-para-la-salud-sobre-la-crisis-sanitaria-del-covid-19/ 
Cruz Páez, F., Ibarra Díaz, C., Rueda Pineda, D., \& Olivares Vera, D. (2020). Análisis exploratorio sobre la apreciación de características predominantes en empresas medianas de México y Colombia en temas de calidad, competitividad, innovación social y productiva. Tendencias, 21(1), 130-156. https://doi.org/10.22267/rtend.202101.130

Cruz Páez, F. O., \& Vanegas Flórez, O. (2020). Vigilancia tecnológica, inteligencia competitiva y cultura organizacional universidad de Cundinamarca Facatativá. Política Globalidad y Ciudadanía, 6(12), 84-101.

Cruz Páez, F., Mera Rodríguez, C., \& Lechuga Cardozo, J. (2019). Evaluación de estrategias de emprendimiento sostenible e innovación implementadas en las unidades productivas del SENA, Centro Industrial y Desarrollo Empresarial de Soacha-Cundinamarca-Colombia. Tendencias, 20(1), 183-202. https://doi.org/10.22267/rtend.192001.113

Darwin, C. (1921). El origen de las especies por medio de la selección natural. (Sin editorial). http://www.traduccionliteraria.org/biblib/D/D1011.pdf

Fernández-Salinero, Carolina., y de la Riva, Beatriz. (2014). Entrepreneurial mentality and culture of entrepreneurship Mentalidad emprendedora y cultura del emprendimiento. Procedia - Social and Behavioral Sciences, 139, 137 - 143. doi: 10.1016/j.sbspro.2014.08.044

Fondo Emprender. (2020). EL FONDO EMPRENDER. Fondo Emprender. http://www.fondoemprender.com/SitePages/Que\%20es\%20FondoEmprender.aspx

Fracica Naranjo, G. (2009). La financiación empresarial y la industria del capital de riesgo: estructura y conceptos. Revista Escuela de Administración de Negocios, (66), 31-65. https://www.redalyc.org/articulo.oa?id=206/20620269003

Gómez, C., Sánchez, V., \& Tovar, G. (2018). Factores endógenos causantes de la permanencia irregular: una lectura desde el actuar docente. Educación Y Humanismo, 20(35), 96-112. https://doi.org/10.17081/eduhum.20.35.3030

Hernández-Perlines, F. (2017). Impacto del aprendizaje del emprendimiento a través de las TIC. International Journal of Educational Research and Innovation (IJERI), 8, 128-147. https://rio.upo.es/xmlui/bitstream/handle/10433/5310/2279-8086-1-PB.pdf?sequence=1\&isAllowed=y

Hernández Sampieri, R., Fernández Collado, C., \& Baptista Lucio, P. (2014). Metodología de la investigación. Mcgraw-hill/ Interamericana editores.

Llano Restrepo, P. (2018). Entrenamiento en el área del servicio al cliente en los centros de experiencia Movistar Cartagena. Conocimiento GIobal, 3(1), $70-82$. http://conocimientoglobal.org/revista/index.php/cglobal/article/view/29

López Pérez, R. (2012). Innovación del modelo de negocio: Propuesta de un modelo Holístico. Madrid. [Tesis doctoral, Universidad Autónoma de Madrid]. Archivo digital. 
https://repositorio.uam.es/bitstream/handle/10486/7825/43365_lopez_perez_ricardo.pdf?sequence=1

Murcia Salazar, H. (2018). Apoyo al área de Proyección Social (CITGO). [Tesis de pregrado, Universidad de Cundinamarca]. Archivo digital. http://repositorio.ucundinamarca.edu.co/bitstream/handle/20.500. 12558/2608/APOYO\%20AL\%20ÁREA\%20DE\%20PROYECCIÓN\%20SOCIAL\%20\%28CITGO\%29\%2 OCENTRO\%20DE\%20INNOVACIÓN\%20TECNOLOGÍA\%20Y\%20GESTIÓN\%20ORGANIZACION.pdf ?sequence $=1 \&$ isAllowed $=y$

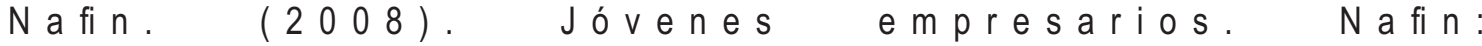
https://www.nafin.com/portalnf/content/financiamiento/jovenes empresarios.html

Santero Sánchez, R., de la Fuente-Cabrero, C. y Laguna Sánchez, P. (2016). Efectos de la crisis sobre la financiación bancaria del emprendimiento. Un análisis de las microempresas españolas desde el sector de las Sociedades de Garantía Recíproca. European Research on Management and Business Economics, 22 (2), 88-93. https://doi.org/10.1016/j.iedee.2015.10.006

Shane, \& Venkataraman. (2000). The promise of entrepreneurship as a field of research. Academy of Management.

Secretaria de Economía. (2010). Programa Nacional de Emprendedores. Secretaria de Economía: http://www.2006-2012.economia.gob.mx/mexico-emprende-en/se-programs/230-programa-nacional-deemprendedores

Valencia Herrera, H. (2015). Complementariedad de las inversiones a largo plazo y de capital de trabajo ante oportunidades de negocios y consideraciones de liquidez en países latinoamericanos. Estudios Gerenciales, 31(137), 364-372. DOI: 10.1016/j.estger.2015.07.004

Vélez Romero, X., \& Ortiz Restrepo, S. (2016). Emprendimiento e innovación: Una aproximación teórica. Ciencias económicas y empresariales. 2 (4), 346-369. 